\title{
Interação zinco e fósforo em solução nutritiva influenciando o crescimento e a produtividade da alface ${ }^{(1)}$
}

\author{
Marialva Alvarenga Moreira(2), Paulo Cezar Rezende Fontes ${ }^{(3)}$ \\ e Maria Isabel de Camargos(2)
}

\begin{abstract}
Resumo - O objetivo deste trabalho foi avaliar o efeito de Zn e P, em solução nutritiva, na absorção desses elementos pela alface (Lactuca sativa L.) e na produtividade dessa hortaliça. O delineamento experimental utilizado foi o de blocos casualizados, com os tratamentos dispostos num esquema fatorial $3 \times 3$, com três repetições. Os tratamentos foram constituídos de três níveis de $\mathrm{Zn}$ : baixo, normal e alto, correspondentes a $0,0,1,5$ e $6,0 \mu \mathrm{M} \mathrm{L}^{-1}$, e de três níveis de $\mathrm{P}$ : baixo, normal e alto, correspondentes a $0,01,1,5$ e $6,0 \mathrm{mM} \mathrm{L}^{-1}$, utilizando-se como fontes o $\mathrm{ZnSO}_{4} \cdot 7 \mathrm{H}_{2} \mathrm{O}$ e o $\mathrm{KH}_{2} \mathrm{PO}_{4}$, respectivamente. Aos 26 dias após o transplante (DAT), a área foliar e a produção de matéria seca da parte aérea não foram influenciadas pelos tratamentos. Aos 52 DAT, a área foliar, o número de folhas e a matéria seca da parte aérea não foram influenciadas pela adição de Zn à solução quando o nível de $\mathrm{P}$ foi baixo. Porém, quando os níveis de $\mathrm{P}$ foram normal e alto, os níveis normal e alto de $\mathrm{Zn}$ propiciaram maiores valores de área foliar, de número de folhas, e de produção de matéria seca da parte aérea. Os teores de $\mathrm{P}$ nas folhas comerciais, em cada nível de $\mathrm{Zn}$, foram semelhantes quando houve adição das doses normal e alta de $\mathrm{P}$ à solução. $\mathrm{O}$ maior teor de $\mathrm{P}$ foi o do caule, seguido do das raízes, e, por último, do das folhas comerciais. Os teores de Zn na matéria seca das folhas comerciais, nos caules e nas raízes, aumentaram com adição de Zn à solução, em todos os níveis de fósforo.
\end{abstract}

Termos para indexação: Lactuca sativa, nutrientes minerais, absorção de nutrientes.

Zinc and phosphorus interaction in nutrient solution affecting lettuce growth and yield

Abstract - The experiment was carried out to evaluate the effect of $\mathrm{Zn}$ and $\mathrm{P}$ levels in nutrient solution on $\mathrm{Zn}$ and $\mathrm{P}$ absorption and lettuce yield (Lactuca sativa L.). A randomized block experimental design in $3 \times 3$ factorial arrangement with three replications was used. The treatments were three $\mathrm{Zn}$ levels: low, normal and high, corresponding to $0.0,1.5$ and $6.0 \mu \mathrm{M} \mathrm{L}^{-1}$ and three P levels: low, normal and high, corresponding to $0.01,1.5$ and $6.0 \mathrm{mM} \mathrm{L}^{-1}$ as $\mathrm{ZnSO}_{4} .7 \mathrm{H}_{2} \mathrm{O}$ and $\mathrm{KH}_{2} \mathrm{PO}_{4}$. Twenty six days after transplantation (DAT), the leaf area and leaf dry weight were not influenced by the treatments. At $52 \mathrm{DAT}$, the leaf area, the leaf number and the shoot dry weight were not affected by $\mathrm{Zn}$ addition when the P level was low; but when P levels were normal and high, the normal and high Zn levels lead to the highest leaf area, leaf number, and shoot dry weight. Phosphorus concentrations in the marketable leaves at each $\mathrm{Zn}$ level were similar at $\mathrm{P}$ normal and high addition levels. The highest $\mathrm{P}$ concentration was observed in the stem followed by roots and marketable leaves. Zinc concentrations in the marketable leaves, stem and roots, increased with $\mathrm{Zn}$ addition to the nutrient solution.

Index terms: Lactuca sativa, mineral nutrients, nutrient uptake.

\section{Introdução}

A alface (Lactuca sativa L.) é a hortaliça folhosa de maior valor comercial no Brasil, e as regiões Sul e

(1) Aceito para publicação em 15 de agosto de 2000 .

(2) Universidade Federal de Viçosa (UFV), Dep. de Fitotecnia, CEP 36571-000 Viçosa, MG

(3) UFV, Dep. de Fitotecnia. Bolsita do CNPq. E-mail pacerefo@mail.ufv.br
Sudeste são as maiores consumidoras. É adaptada a clima ameno, sendo própria para cultivos de outono/ inverno. No verão, a redução na relação entre oferta e procura e a baixa qualidade do produto, geralmente, proporcionam maiores cotações da alface.

Por outro lado, o uso da cobertura plástica tem viabilizado a produção de alface durante todo o ano, protegendo as plantas contra danos provocados por chuvas, ventos fortes e incidência direta da radiação solar em regiões tropicais e subtropicais, e prote- 
gendo contra a geada, em regiões de inverno muito frio. Além da plasticultura, a hidroponia tem sido utilizada com sucesso em plantios de alface, pois possibilita elevar a produtividade, aumentar o número de colheitas por ano sem a necessidade de rotação da cultura, reduzir os gastos com defensivos agrícolas, obter um produto comercial de melhor aspecto, além de ocupar menor espaço físico para o cultivo (Castellane \& Araújo, 1995). Apesar do aumento do uso da hidroponia no País, há carência de resultados experimentais sobre o efeito das concentrações dos nutrientes em solução para o cultivo da alface.

Concentrações dos nutrientes na solução nutritiva utilizada para a produção de alface afetam a produtividade da cultura, quase sempre devido ao antagonismo iônico. Isto é, na solução nutritiva, cujo poder-tampão é quase nulo, alta concentração de um elemento pode diminuir a absorção de outro, acarretando deficiência e afetando o crescimento das plantas (Rengel \& Granham, 1995). Tem sido observado que alta concentração de $\mathrm{P}$ pode induzir ou aumentar a deficiência de Zn, particularmente em condições de baixa disponibilidade deste (Cakmak \& Marschner, 1987). Isto pode ser devido ao excesso de $\mathrm{P}$ aumentar o requerimento fisiológico de $\mathrm{Zn}$ (Webb \& Loneragan, 1988), reduzir as taxas de crescimento radicular e de transporte do $\mathrm{Zn}$ da raiz para a parte aérea, ou pela presença do cátion acompanhante da fonte fosfatada, o qual pode inibir a absorção de Zn (Loneragan \& Webb, 1993).

O presente trabalho teve como objetivo avaliar o efeito de níveis de $\mathrm{Zn}$ e de $\mathrm{P}$ em solução nutritiva na absorção desses elementos pela alface e na produtividade dessa hortaliça.

\section{Material e Métodos}

O experimento foi realizado em casa de vegetação, no campus da Universidade Federal de Viçosa, em vasos de 8 litros com solução nutritiva aerada. O delineamento experimental foi o de blocos casualizados, com os tratamentos dispostos num esquema fatorial $3 \times 3$, com três repetições. Os tratamentos foram constituídos de três níveis de Zn: baixo ou sem adição direta, normal e alto, correspondentes a $0,0,1,5$ e $6,0 \mu \mathrm{M} \mathrm{L}^{-1}$, e de três níveis de P: baixo, normal e alto, correspondentes a $0,01,1,5$ e $6,0 \mathrm{mM} \mathrm{L}^{-1}$, sendo utilizadas como fontes o $\mathrm{ZnSO}_{4} \cdot 7 \mathrm{H}_{2} \mathrm{O}$ e o $\mathrm{KH}_{2} \mathrm{PO}_{4}$. Os níveis de $\mathrm{Zn}$ e $\mathrm{P}$ considerados normais foram baseados na recomendação de Resh (1997).

A semeadura da alface, cultivar Regina, ocorreu no dia 14 de julho de 1997, em areia purificada com ácido clorídri- co, segundo os procedimentos de Fontes (1986). Após a emergência das plantas, o substrato foi irrigado com solução de Steiner (Steiner, 1984) modificada e contendo $1 / 2$ das concentrações originais dos macro e micronutrientes, porém sem zinco. A solução modificada continha as seguintes concentrações, em mmol L-1, de macronutrientes: 8,4 de $\mathrm{N}_{-\mathrm{NO}_{3}^{-}}^{-} ; 3,6$ de N-NH $4_{4}^{+} ; 7,0$ de $\mathrm{K}^{+} ; 4,5 \mathrm{de} \mathrm{Ca}^{2+} ; 2,0$ de $\mathrm{Mg}^{2+}$ e 3,5 de S-SO ${ }_{4}^{2-}$, utilizando-se $\mathrm{Ca}\left(\mathrm{NO}_{3}\right)_{2} \cdot 4 \mathrm{H}_{2} \mathrm{O}$, $\left(\mathrm{NH}_{4}\right)_{2} \mathrm{SO}_{4}, \mathrm{KCl}$ e $\mathrm{MgSO}_{4} \cdot 7 \mathrm{H}_{2} \mathrm{O}$. Quanto aos micronutrientes, foram utilizadas as seguintes concentrações, expressas em $\mu \mathrm{mol} \mathrm{L}^{-1}: 46,0$ de B; 0,3 de Cu; 45 de $\mathrm{Fe} ; 35$ de $\mathrm{Mn}$ e 0,5 de $\mathrm{Mo}$, utilizando-se $\mathrm{H}_{3} \mathrm{BO}_{3}$, $\mathrm{CuSO}_{4} \cdot 5 \mathrm{H}_{2} \mathrm{O}, \quad \mathrm{FeCl}_{3} \cdot 6 \mathrm{H}_{2} \mathrm{O}, \quad \mathrm{MnSO}_{4} \cdot \mathrm{H}_{2} \mathrm{O}$ e $\left(\mathrm{NH}_{4}\right)_{6} \mathrm{Mo}_{7} \mathrm{O}_{24} \cdot 4 \mathrm{H}_{2} \mathrm{O}$, sendo todos sais p.a. e água deionizada. As soluções estoques de $\mathrm{N}, \mathrm{P}, \mathrm{K}, \mathrm{Ca}$ e $\mathrm{Mg}$ foram submetidas a técnica de purificação para a remoção do $\mathrm{Zn}$, com o reagente pirrolidina ditiocarbamato de amônio, usando como solvente o clorofórmio (Fontes, 1986). Dentre os micronutrientes, somente o cloreto férrico foi purificado pela resina de troca aniônica (Fontes, 1986). Antes de ocorrerem as diluições das soluções estoque submetidas ao processo de purificação, estas foram aquecidas, para eliminar o clorofórmio remanescente.

O transplantio das mudas para os vasos contendo os tratamentos ocorreu no dia 4 de agosto de 1997. Nesta data, as mudas estavam com $5 \mathrm{~cm}$ de altura, 4 a 6 folhas, tendo sido colocadas duas por vaso. Os vasos utilizados na condução do experimento foram previamente lavados com $\mathrm{HCl}$ 1:1 e, em seguida, lavados com água deionizada. $\mathrm{O} \mathrm{pH}$ da solução nutritiva foi mantido em 6,0, por correção diária adicionando-se $\mathrm{HCl} 1 \mathrm{~N}$ ou $\mathrm{NaOH} 1 \mathrm{~N}$. O nível da solução, em cada vaso, foi de $1,5 \mathrm{~cm}$ da borda superior, controlado pelo acréscimo de água deionizada, sendo aerada continuamente.

Após 26 dias do transplante (DAT), quando o K atingiu $70 \%$ da concentração original, houve a troca da solução nutritiva de todos os vasos. Nesta data, foi retirada uma das plantas de cada vaso, para a determinação da área foliar e do peso da matéria seca da parte aérea. Após 52 dias do transplante (DAT), as plantas remanescentes foram colhidas. Imediatamente foi feita a avaliação visual da parte aérea e das raízes, sendo em seguida separadas em folhas comerciais e não-comerciais, caule e raízes. Nesta ocasião, foram determinados área foliar, número de folhas e peso da matéria seca das partes amostradas. Em seguida, os tecidos foram lavados com água deionizada e secadas em estufa a $80^{\circ} \mathrm{C}$; posteriormente, foram moídos e submetidos a digestão nitricoperclórica. No extrato nitricoperclórico foi determinado o teor de $\mathrm{P}$ por espectrofotometria do azul de molibdênio (Braga \& Defelipo, 1974) e de Zn por espectrofotometria de absorção atômica (Association of Official Analytical Chemists, 1975).

Os resultados foram submetidos a análise de variância e, independentemente da significância da interação, os efeitos dos níveis de $\mathrm{P}$ foram avaliados em cada nível de $\mathrm{Zn}$, e as médias, comparadas pelo teste de Tukey a 5\% de probabilidade. 


\section{Resultados e Discussão}

Aos 52 DAT, a área foliar não foi afetada pela adição direta de $\mathrm{Zn}$ à solução quando o nível de $\mathrm{P}$ foi baixo; mas, quando os níveis de $\mathrm{P}$ foram normal e alto, os níveis normal e alto de $\mathrm{Zn}$ propiciaram as maiores áreas foliares (Tabela 1). Os níveis baixo, normal e alto de P na solução não afetaram a área foliar quando o nível de $\mathrm{Zn}$ foi baixo; porém, nos níveis normal e alto de $Z n$, a adição do $P$ promoveu aumento da área foliar. De modo semelhante, o número de folhas e a produção de matéria seca da parte aérea não foram afetados pela adição de Zn à solução, quando o nível de $\mathrm{P}$ foi baixo; mas, quando o nível de $\mathrm{P}$ foi baixo e os níveis de Zn baixo, normal ou alto, o número de folhas e produção de matéria seca da parte aérea diminuíram.

A produção de matéria seca de raízes diminuiu com a adição de $\mathrm{Zn}$ à solução quando o nível de $\mathrm{P}$ foi baixo; porém, nos níveis normal e alto de $\mathrm{P}$ não houve efeito da adição de $\mathrm{Zn}$ à solução (Tabela 1 ). Nos níveis baixo e normal de $\mathrm{Zn}$, a adição de $\mathrm{P}$ não afetou a produção de matéria seca da raiz; mas, no nível alto de $\mathrm{Zn}$, a maior produção de matéria seca da raiz ocorreu no nível alto de $\mathrm{P}$.

Com relação à planta inteira, no nível baixo de $\mathrm{P}$ não houve efeito da adição de $\mathrm{Zn}$ à solução; no nível normal de $\mathrm{P}$ a adição de $\mathrm{Zn}$ proporcionou maior produção de matéria seca, e no nível alto de $\mathrm{P}$, os níveis normal e alto de $\mathrm{Zn}$ proporcionaram maiores produções de matéria seca (Tabela 1). O maior valor do peso de matéria seca da planta inteira foi $18,17 \mathrm{~g}$, correspondente a $412 \mathrm{~g}$ de peso verde encontrado em níveis alto de $\mathrm{P}$ e normal de $\mathrm{Zn}$. Costa (1998) encontrou, na cultivar Regina, aos 41 DAT, em solo adubado com composto orgânico de lixo urbano, 19,64 g de matéria seca da parte aérea por planta, e Delistoianov (1997), em meio hidropônico, obteve em média 5,85 g de matéria seca de folhas comerciais por planta. Efeitos do Zn e do P sobre a área foliar e a produção de matéria seca da parte aérea e da raiz de alface, em meio hidropônico, não foram encontrados por Iorio et al. (1996).

Dependendo dos tratamentos utilizados, as plantas apresentaram diferentes sintomas visuais na parte aérea e nas raízes. Com o nível baixo de $\mathrm{P}$ e os níveis baixo, normal e alto de Zn na solução, as plantas foram pouco desenvolvidas, apresentando escurecimento das raízes, e as folhas mais velhas com clorose e necrose nos bordos. Garcia (1982) observou que as plantas crescidas em solução nutritiva sem $\mathrm{P}$ apresentavam desenvolvimento afetado, com sistema radicular bem desenvolvido mas de coloração escura, acinzentada.

Nos níveis normal e alto de $\mathrm{P}$ e baixo de $\mathrm{Zn}$, as plantas apresentavam pequeno desenvolvimento, com as raízes claras e as folhas mais velhas com

Tabela 1. Área foliar, número de folhas e peso da matéria seca da parte aérea, raiz e planta inteira de alface, cultivada durante 52 dias em hidroponia, em razão dos níveis baixo, normal e alto de zinco e de fósforo na solução nutritiva ${ }^{(1)}$

\begin{tabular}{|c|c|c|c|c|c|c|}
\hline \multirow{2}{*}{$\begin{array}{c}\text { Zn na } \\
\text { solução }\end{array}$} & \multirow{2}{*}{$\begin{array}{c}\text { P na } \\
\text { solução }\end{array}$} & \multirow{2}{*}{$\begin{array}{l}\text { Área } \\
\text { foliar }\end{array}$} & \multirow{2}{*}{$\begin{array}{l}\text { Número de } \\
\text { folhas }\end{array}$} & \multicolumn{3}{|c|}{ Matéria seca } \\
\hline & & & & $\begin{array}{l}\text { Parte } \\
\text { aérea }^{(2)}\end{array}$ & Raiz & Planta inteira \\
\hline & & $\left(\mathrm{cm}^{2}\right.$ planta $\left.^{-1}\right)$ & & & (g planta & ------------ \\
\hline \multirow{4}{*}{ Baixo } & Baixo & $1.936 \mathrm{Aa}$ & $13 \mathrm{Ab}$ & $5,08 \mathrm{Ab}$ & $1,88 \mathrm{Aa}$ & 6,96Аa \\
\hline & Normal & $3.759 \mathrm{Ba}$ & $22 \mathrm{Ba}$ & $8,23 \mathrm{Ba}$ & $1,48 \mathrm{Aa}$ & $9,71 \mathrm{Ba}$ \\
\hline & Alto & $3.219 \mathrm{Ba}$ & $17 \mathrm{Cab}$ & $8,32 \mathrm{Ba}$ & $1,28 \mathrm{Aa}$ & $9,60 \mathrm{Ba}$ \\
\hline & Média & 2.971 & 17 & 7,21 & 1,54 & 8,76 \\
\hline \multirow[t]{4}{*}{ Normal } & Baixo & $2.182 \mathrm{Ab}$ & $17 \mathrm{Ab}$ & $5,70 \mathrm{Ac}$ & $1,99 \mathrm{Aa}$ & $7,69 \mathrm{Ab}$ \\
\hline & Normal & 4.966ABab & $42 \mathrm{Aa}$ & $9,01 \mathrm{Bb}$ & $1,26 \mathrm{Aa}$ & $10,27 \mathrm{Bb}$ \\
\hline & Alto & 7.383Aa & $43 \mathrm{Aa}$ & $16,64 \mathrm{Aa}$ & $1,53 \mathrm{Aa}$ & $18,17 \mathrm{Aa}$ \\
\hline & Média & 4.843 & 34 & 10,45 & 1,59 & 12,04 \\
\hline \multirow[t]{4}{*}{ Alto } & Baixo & $2.669 \mathrm{Ab}$ & $19 \mathrm{Ab}$ & $7,02 \mathrm{Ab}$ & $0,67 \mathrm{Bb}$ & 7,69Ab \\
\hline & Normal & $8.489 \mathrm{Aa}$ & $38 \mathrm{Aa}$ & $14,63 \mathrm{Aa}$ & $1,20 \mathrm{Aab}$ & $15,83 \mathrm{Aa}$ \\
\hline & Alto & 7.073ABa & $34 \mathrm{Ba}$ & $14,61 \mathrm{Aa}$ & $1,73 \mathrm{Aa}$ & $16,34 \mathrm{Aa}$ \\
\hline & Média & 6.077 & 30 & 12,09 & 1,20 & 13,29 \\
\hline
\end{tabular}

${ }^{(1) L e t r a s ~ m a i u ́ s c u l a s ~ c o m p a r a m ~ o s ~ n i ́ v e i s ~ d e ~} \mathrm{Zn}$ em cada nível de $\mathrm{P}$, e letras minúsculas, os níveis de $\mathrm{P}$ em cada nível de Zn; em cada coluna, as médias seguidas pela mesma letra maiúscula ou minúscula não expressam diferenças significativas a $5 \%$ de probabilidade, pelo teste de Tukey. ${ }^{(2)}$ Soma do peso da matéria seca de caule e de folhas comercial e não-comercial. 
clorose nos bordos. Nos níveis normal e alto de $\mathrm{P}$ e de $\mathrm{Zn}$ as plantas estavam bem desenvolvidas, com raízes claras e as folhas sem necrose ou clorose.

No nível baixo de $\mathrm{Zn}$, as raízes permaneceram escuras, e as folhas apresentavam áreas coriáceas, necrose nos bordos, menor área foliar, e menor número de folhas. A presença de clorose e necrose em folhas velhas de plantas deficientes em Zn são usualmente efeitos secundários, muitas vezes, em decorrência da toxidez do P (Marschner, 1995), pois o Zn é importante para o crescimento (Grewal et al., 1997) e para manutenção da integridade da membrana plasmática da raiz (Cakmak \& Marschner, 1988; Welch \& Norvell, 1993). Em condição de deficiência de $\mathrm{Zn}$ pode haver significativo aumento na absorção de $\mathrm{P}$, com conseqüente toxidez na parte aérea das plantas (Marschner \& Cakmak, 1986; Parker, 1997).

Os teores de $\mathrm{P}$ nos diversos órgãos da planta, em cada nível de $\mathrm{Zn}$, aumentaram com a presença de $\mathrm{P}$, mas foram semelhantes nos níveis normal e alto de $\mathrm{P}$ (Tabela 2). De maneira geral, os teores médios de $\mathrm{P}$ foram maiores no caule, seguidos das raízes, e por último, das folhas comerciais, atingindo os valores médios de 1,09, 0,95 e 0,65 dag kg-1 de matéria seca, respectivamente. Furlani et al. (1978) encontraram, como valor médio de $\mathrm{P}, 0,77$ dag $\mathrm{kg}^{-1}$ de matéria seca em folhas de alface; Garcia (1982) encontrou valores entre 0,67 e $0,92 \mathrm{dag} \mathrm{kg}^{-1}$, e Delistoianov (1997) 0,78 dag $\mathrm{kg}^{-1}$. A maior concentração de P nas folhas comerciais (1,24 dag $\mathrm{kg}^{-1}$ de matéria seca) foi observada quando o nível de $\mathrm{P}$ foi alto e o de $\mathrm{Zn}$ baixo. Provavelmente, a limitação de $\mathrm{Zn}$ causou danos ao sistema de membranas das raízes, permitindo o acúmulo de $\mathrm{P}$ nas folhas, conforme verificado por outros autores (Cakmak \& Marschner, 1988), possivelmente em razão da taxa de absorção ter sido maior que a taxa de produção de matéria seca. Por outro lado, quando o nível de $\mathrm{P}$ na solução foi alto e os níveis de $\mathrm{Zn}$ foram normal ou alto, o teor de $\mathrm{P}$ nas folhas diminuiu (Tabela 2), e o oposto foi verificado com a quantidade de $\mathrm{P}$ acumulada na parte aérea (Tabela 3). Tal fato pode ser devido ao aumento do metabolismo de P pela planta (Iorio et al., 1996), além da sua diluição em maiores área foliar e matéria seca da parte aérea (Tabela 1).

$\mathrm{O}$ conteúdo de $\mathrm{P}$ nas folhas comerciais não foi afetado pela adição de Zn à solução, quando o nível de $\mathrm{P}$ foi baixo (Tabela 3). Em valor absoluto, o maior conteúdo de $\mathrm{P}$ na matéria seca da planta foi observado no tratamento $\mathrm{Zn}$ normal e $\mathrm{P}$ alto, e correspondeu a 166 dag planta $^{-1}$. Deste total, 57, 17, 15 e 11\% foram acumulados nas folhas comerciais, caule, raízes e folhas não-comerciais, respectivamente.

Os teores de $\mathrm{Zn}$ nas folhas comerciais aumentaram com a adição de $Z n$ à solução nutritiva (Tabela 4). No nível alto de $\mathrm{Zn}$, os teores de $\mathrm{Zn}$ nas folhas comerciais diminuíram com adição dos níveis normal e alto de $\mathrm{P}$ à solução. Também, em batata, o teor de $\mathrm{Zn}$ nas folhas foi influenciado pela interação entre Zn e P (Christensen \& Jackson, 1981), sendo aumentado pela suplementação de Zn (Moreira, 1998). Os teores médios de $\mathrm{Zn}$ foram maiores nas folhas comerciais, e em seguida, nas raízes, e por último, nos caules (Tabela 4), atingindo os valores médios de 64, 59 e $42 \mathrm{mg} \mathrm{kg}^{-1}$ de matéria seca, respectivamente. Santos et al. (1998) encontraram valor de $\mathrm{Zn}$ de $62 \mathrm{mg} \mathrm{kg}^{-1}$ de matéria seca de folhas de alface cultivadas no solo; também em folhas, Furlani et al. (1978) encontraram 94 a $116 \mathrm{mg} \mathrm{kg}^{-1}$, e Garcia et al. (1982) observaram variação na concentração de Zn em decorrência da idade da planta e da cultivar.

Quando o nível de $\mathrm{Zn}$ foi baixo ou normal, o conteúdo de $Z$ n nas raízes não foi afetado pela adição de $\mathrm{P}$; todavia, no nível alto de $\mathrm{Zn}$, a adição de $\mathrm{P}$ aumentou o conteúdo de $\mathrm{Zn}$ na raiz (Tabela 5).

Tabela 2. Teor de P na matéria seca das folhas comerciais, caule e raiz de alface, cultivada durante 52 dias em hidroponia, em razão dos níveis baixo, normal e alto de $\mathrm{Zn}$ e de P na solução nutritiva ${ }^{(1)}$

\begin{tabular}{|c|c|c|c|c|}
\hline \multirow{2}{*}{$\begin{array}{c}\text { Zn na } \\
\text { solução }\end{array}$} & \multirow{2}{*}{$\begin{array}{c}\text { P na } \\
\text { solução }\end{array}$} & \multicolumn{3}{|c|}{ Teor de P na matéria seca } \\
\hline & & $\begin{array}{c}\text { Folhas } \\
\text { comerciais }\end{array}$ & Caule & Raiz \\
\hline \multirow{4}{*}{ Baixo } & & ------------- & $\left.\mathrm{ag} \mathrm{kg}^{-1}\right)$ & \\
\hline & Baixo & $0,10 \mathrm{Ab}$ & $0,07 \mathrm{Ab}$ & $0,17 \mathrm{Ab}$ \\
\hline & Normal & $1,14 \mathrm{Aa}$ & $1,75 \mathrm{Aa}$ & $0,84 \mathrm{Ba}$ \\
\hline & Alto & $1,24 \mathrm{Aa}$ & $1,76 \mathrm{Aa}$ & $0,89 \mathrm{Ba}$ \\
\hline \multirow[t]{3}{*}{ Normal } & Baixo & $0,11 \mathrm{Ab}$ & $0,09 \mathrm{Ab}$ & $0,18 \mathrm{Ab}$ \\
\hline & Normal & $0,80 \mathrm{Ba}$ & $1,53 \mathrm{ABa}$ & $1,54 \mathrm{Aa}$ \\
\hline & Alto & $0,74 \mathrm{Ba}$ & $1,56 \mathrm{ABa}$ & $1,65 \mathrm{Aa}$ \\
\hline \multirow[t]{3}{*}{ Alto } & $\overline{\text { Baixo }}$ & $0,13 \mathrm{Ab}$ & $0,16 \mathrm{Ab}$ & $0,21 \mathrm{Ab}$ \\
\hline & Normal & $0,75 \mathrm{Ba}$ & $1,45 \mathrm{Ba}$ & $1,43 \mathrm{Aa}$ \\
\hline & Alto & $0,80 \mathrm{Ba}$ & $1,46 \mathrm{Ba}$ & $1,63 \mathrm{Aa}$ \\
\hline
\end{tabular}

(1)Letras maiúsculas comparam os níveis de $\mathrm{Zn}$ em cada nível de $\mathrm{P}$, e letras minúsculas, os níveis de $\mathrm{P}$ em cada nível de $\mathrm{Zn}$; em cada coluna, as médias seguidas pela mesma letra maiúscula ou minúscula não expressam diferenças significativas a $5 \%$ de probabilidade, pelo teste de Tukey. 
Tabela 3. Conteúdo de P na matéria seca das folhas comerciais e não-comerciais, caule, raízes e planta inteira de alface, cultivada durante 52 dias em hidroponia, em razão dos níveis baixo, normal e alto de $\mathrm{Zn}$ e de $\mathrm{P}$ na solução nutritiva ${ }^{(1)}$.

\begin{tabular}{|c|c|c|c|c|c|c|}
\hline \multirow{2}{*}{$\begin{array}{c}\text { Zn na } \\
\text { solução }\end{array}$} & \multirow{2}{*}{$\begin{array}{c}\text { P na } \\
\text { solução }\end{array}$} & \multicolumn{5}{|c|}{ Conteúdo de P na matéria seca } \\
\hline & & $\begin{array}{c}\text { Folhas } \\
\text { comerciais }\end{array}$ & $\begin{array}{l}\text { Folhas-não } \\
\text { comerciais }\end{array}$ & Caule & Raiz & $\begin{array}{l}\text { Planta } \\
\text { inteira }\end{array}$ \\
\hline \multirow{4}{*}{ Baixo } & & ------------- & & -(dag pla & & \\
\hline & Baixo & $1,91 \mathrm{Ab}$ & $2,51 \mathrm{Ab}$ & $0,46 \mathrm{Ab}$ & $3,21 \mathrm{Ab}$ & $8,09 \mathrm{Ab}$ \\
\hline & Normal & $45,19 \mathrm{Ba}$ & $51,36 \mathrm{Aa}$ & $21,70 \mathrm{Aa}$ & $12,45 \mathrm{Aa}$ & $130,70 \mathrm{ABa}$ \\
\hline & Alto & $30,60 \mathrm{Ba}$ & $62,01 \mathrm{Aa}$ & $24,48 \mathrm{Aa}$ & $11,55 \mathrm{Ba}$ & $128,64 \mathrm{Ba}$ \\
\hline \multirow[t]{3}{*}{ Normal } & Baixo & $2,94 \mathrm{Ac}$ & $2,64 \mathrm{Aa}$ & $0,61 \mathrm{Ab}$ & $3,53 \mathrm{Ab}$ & $9,72 \mathrm{Ac}$ \\
\hline & Normal & $45,44 \mathrm{Bb}$ & $12,85 \mathrm{Ba}$ & $24,39 \mathrm{Aa}$ & $19,58 \mathrm{Aa}$ & $102,26 \mathrm{Bb}$ \\
\hline & Alto & $93,40 \mathrm{Aa}$ & $18,06 \mathrm{Ba}$ & $28,90 \mathrm{Aa}$ & $25,17 \mathrm{Aa}$ & $165,53 \mathrm{Aa}$ \\
\hline \multirow[t]{3}{*}{ Alto } & Baixo & $5,92 \mathrm{Ab}$ & $5,23 \mathrm{Aa}$ & $1,11 \mathrm{Ab}$ & $3,93 \mathrm{Ab}$ & $16,19 \mathrm{Ab}$ \\
\hline & Normal & $75,76 \mathrm{Aa}$ & $17,38 \mathrm{Ba}$ & $25,36 \mathrm{Aa}$ & $17,38 \mathrm{Aa}$ & $135,88 \mathrm{Aa}$ \\
\hline & Alto & $79,78 \mathrm{Aa}$ & $18,03 \mathrm{Ba}$ & $30,44 \mathrm{Aa}$ & $20,42 \mathrm{Aa}$ & $148,67 \mathrm{ABa}$ \\
\hline
\end{tabular}

(1)Letras maiúsculas comparam os níveis de $\mathrm{Zn}$ em cada nível de $\mathrm{P}$, e letras minúsculas, os níveis de $\mathrm{P}$ em cada nível de $\mathrm{Zn}$; em cada coluna, as médias seguidas pela mesma letra maiúscula ou minúscula não expressam diferenças significativas a $5 \%$ de probabilidade, pelo teste de Tukey.

Tabela 4. Teor de Zn na matéria seca das folhas comerciais, caule e raiz de alface, cultivada durante 52 dias em hidroponia, em razão dos níveis baixo, normal e alto de $\mathrm{Zn}$ e de P na solução nutritiva ${ }^{(1)}$

\begin{tabular}{|c|c|c|c|c|}
\hline \multirow{2}{*}{$\begin{array}{c}\text { Zn na } \\
\text { solução }\end{array}$} & \multirow{2}{*}{$\begin{array}{c}\text { P na } \\
\text { solução }\end{array}$} & \multicolumn{3}{|c|}{ Teor de zinco na matéria seca } \\
\hline & & $\begin{array}{c}\text { Folhas } \\
\text { comerciais }\end{array}$ & Caule & Raiz \\
\hline \multirow{4}{*}{ Baixo } & & $---------(c$ & $\left.\mathrm{g} \mathrm{kg}^{-1}\right)$ & \\
\hline & Baixo & $24 \mathrm{Ca}$ & $12 \mathrm{Bb}$ & $18 \mathrm{Ba}$ \\
\hline & Normal & $15 \mathrm{Ba}$ & $35 \mathrm{Ca}$ & $17 \mathrm{Ca}$ \\
\hline & Alto & $14 \mathrm{Ca}$ & $17 \mathrm{Cb}$ & $15 \mathrm{Ca}$ \\
\hline \multirow[t]{3}{*}{ Normal } & Baixo & $63 \mathrm{Ba}$ & $33 \mathrm{Ab}$ & $38 \mathrm{ABa}$ \\
\hline & Normal & 58Aa & $52 \mathrm{Ba}$ & $49 \mathrm{Ba}$ \\
\hline & Alto & $51 \mathrm{Ba}$ & $54 \mathrm{Ba}$ & $56 \mathrm{Ba}$ \\
\hline \multirow[t]{3}{*}{ Alto } & Baixo & $198 \mathrm{Aa}$ & $37 \mathrm{Ab}$ & $64 \mathrm{Ab}$ \\
\hline & Normal & $72 \mathrm{Ab}$ & $68 \mathrm{Aa}$ & $86 \mathrm{Ab}$ \\
\hline & Alto & $78 \mathrm{Ab}$ & 77Aa & 191Aa \\
\hline
\end{tabular}

${ }^{(1)}$ Letras maiúsculas comparam os níveis de Zn em cada nível de $\mathrm{P}$, e letras minúsculas, os níveis de $\mathrm{P}$ em cada nível de $\mathrm{Zn}$; em cada coluna, as médias seguidas pela mesma letra maiúscula ou minúscula não expressam diferenças significativas a $5 \%$ de probabilidade, pelo teste de Tukey.

Nas condições de alta suplementação de Zn, o P pode imobilizar o $\mathrm{Zn}$ nas raízes, por meio da formação de fitato de $\mathrm{Zn}$, o qual tem sido observado que ocorre na grande maioria das plantas (Loneragan \& Webb, 1993). A adição de Zn à solução aumentou o conteúdo de Zn na planta inteira, independentemente do nível de $\mathrm{P}$; porém, nos níveis de $\mathrm{Zn}$ baixo ou alto, a adição de $\mathrm{P}$ à solução não afetou os conteúdos de Zn (Tabela 5). Em valor absoluto, o maior conteúdo de Zn na planta inteira foi observado nos tratamentos $\mathrm{Zn}$ e P altos (Tabela 5), e correspondeu a
Tabela 5. Conteúdo de Zn na matéria seca das folhas comerciais e não-comerciais, caule, raízes e planta inteira de alface, cultivada durante 52 dias em hidroponia, em razão dos níveis baixo, normal e alto de $\mathrm{Zn}$ e de $\mathrm{P}$ na solução nutritiva $^{(1)}$

\begin{tabular}{|c|c|c|c|c|c|c|}
\hline \multirow{2}{*}{$\begin{array}{c}\text { Zn na } \\
\text { solução }\end{array}$} & \multirow{2}{*}{$\begin{array}{l}\text { P na } \\
\text { solução }\end{array}$} & \multicolumn{5}{|c|}{ Conteúdo de Zn na matéria seca } \\
\hline & & $\begin{array}{c}\text { Folhas } \\
\text { comerciais }\end{array}$ & $\begin{array}{l}\text { Folhas-não } \\
\text { comerciais }\end{array}$ & Caule & Raiz & $\begin{array}{l}\text { Planta } \\
\text { inteira }\end{array}$ \\
\hline & & 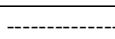 & $-(\mu \mathrm{g} \mathrm{p}$ & anta $\left.^{-1}\right)$ & 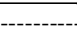 & \\
\hline \multirow[t]{3}{*}{ Baixo } & Baixo & $47 \mathrm{Ba}$ & $52 \mathrm{Ba}$ & $7 \mathrm{Aa}$ & $33 \mathrm{Aa}$ & 139Ba \\
\hline & Normal & $51 \mathrm{Ba}$ & $66 \mathrm{Ba}$ & $44 \mathrm{Ba}$ & $25 \mathrm{Aa}$ & $186 \mathrm{Ba}$ \\
\hline & Alto & $33 \mathrm{Ba}$ & $65 \mathrm{Ba}$ & $25 \mathrm{Ca}$ & $18 \mathrm{Ba}$ & $141 \mathrm{Ca}$ \\
\hline \multirow[t]{3}{*}{ Normal } & Baixo & $165 \mathrm{Bb}$ & $148 \mathrm{Ba}$ & $21 \mathrm{Ab}$ & $74 \mathrm{Aa}$ & $408 \mathrm{Bb}$ \\
\hline & Normal & $328 \mathrm{Bab}$ & $113 \mathrm{Ba}$ & $83 \mathrm{Aa}$ & $61 \mathrm{Aa}$ & $585 \mathrm{Bab}$ \\
\hline & Alto & $661 \mathrm{Aa}$ & $125 \mathrm{Ba}$ & $100 \mathrm{Ba}$ & $86 \mathrm{Ba}$ & $972 \mathrm{Ba}$ \\
\hline \multirow[t]{3}{*}{$\overline{\text { Alto }}$} & Baixo & 798Aa & $473 \mathrm{Aa}$ & $35 \mathrm{Ac}$ & $40 \mathrm{Ab}$ & $1.346 \mathrm{Aa}$ \\
\hline & Normal & 759Aa & $435 \mathrm{Aa}$ & $118 \mathrm{Ab}$ & $106 \mathrm{Ab}$ & $1.418 \mathrm{Aa}$ \\
\hline & Alto & $762 \mathrm{Aa}$ & 494Aa & $160 \mathrm{Aa}$ & 356Aa & $1.772 \mathrm{Aa}$ \\
\hline
\end{tabular}

${ }^{(1)} L$ tras maiúsculas comparam os níveis de $\mathrm{Zn}$ em cada nível de $\mathrm{P}$, e letras minúsculas, os níveis de $\mathrm{P}$ em cada nível de Zn; em cada coluna, as médias seguidas pela mesma letra maiúscula ou minúscula não expressam diferenças significativas a $5 \%$ de probabilidade, pelo teste de Tukey.

$1.772 \mu$ g planta $^{-1}$. Destes, $43,20,9$ e $28 \%$ foram absorvidos pelas folhas comerciais, raízes, caule e folhas não-comerciais, respectivamente.

\section{Conclusões}

1. Quando o nível de P na solução é baixo, não há efeito de níveis de $\mathrm{Zn}$ sobre a produtividade da alface.

2. A produção de matéria seca total da alface é afetada pela adição de $\mathrm{Zn}$ à solução, quando o nível de $\mathrm{P}$ presente é normal ou alto. 
3. As maiores produtividades e absorção de $\mathrm{P}$ são alcançadas quando a solução contém nível alto de $\mathrm{P}$ e nível normal de $\mathrm{Zn}$.

4. Níveis de Zn na solução não têm efeito sobre a absorção de $\mathrm{P}$ pela planta, quando o nível de $\mathrm{P}$ na solução é baixo.

5. Os maiores teores de P estão no caule, seguindo-se, em ordem decrescente, nas raízes e folhas comerciais.

6. Independentemente do nível de P na solução, a maior absorção de $\mathrm{Zn}$ ocorre no nível alto de $\mathrm{Zn}$.

7. Os teores de $Z n$ nas folhas comerciais, no nível alto de $\mathrm{Zn}$ na solução, diminuem com a adição de $\mathrm{P}$ à solução.

\section{Referências}

ASSOCIATION OF OFFICIAL ANALYTICAL CHEMISTS (Gaithersburg, Estados Unidos). Official methods of analysis of association of official analytical. 12. ed. Washington, 1975. $1094 \mathrm{p}$.

BRAGA, J. M.; DEFELIPO, B. V. Determinação espectrofotométrica de fósforo em extratos de solos e material vegetal. Revista Ceres, Viçosa, v. 21, n. 113, p. 73-85, 1974.

CAKMAK, I.; MARSCHNER, H. Increase in membrane permeability and exudation in roots of zinc deficient plants. Journal of Plant Physiology, Jena, v. 132, n. 3, p. 356361,1988

CAKMAK, I.; MARSCHNER, H. Mechanism of phosphorus-induced zinc deficiency in cotton. III. Changes in physiological availability of zinc in plants. Physiologia Plantarum, Copenhagen, v. 70, n. 1, p. 13-20, 1987.

CASTEllane, P. D.; ARAÚJO, J. A. C. Cultivo sem solo: hidroponia. Jaboticabal : FUNEP, 1995. 43 p.

CHRISTENSEN, N. W.; JACKSON, T. L. Potential for phosphorus toxicity in $\mathrm{Zn}$ stressed corn and potato. Soil Science Society of America Journal, Madison, v. 45, n. 5, p. 904-909, 1981.

COSTA, C. A. Produção de alface em cultivos sucessivos adubados com composto orgânico de lixo urbano e teor de metais pesados no solo e na planta. Viçosa : UFV, 1998. 77 p. Dissertação de Mestrado.
DELISTOIANOV, F. Produção, teores de nitrato e capacidade de rebrota de cultivares de alface, sob estufa, em hidroponia e solo, no verão e outono. Viçosa : UFV, 1997. 76 p. Dissertação de Mestrado.

FONTES, R. L. F. Purificação de soluções nutritivas para indução de deficiência de zinco e cobre, em plantas. Viçosa : UFV, 1986. 72 p. Dissertação de Mestrado.

FURLANI, A. M. C.; FURLANI, P. R.; BATAGLIA, R.; HIROCE, J. R.; GALLO, J. R. Composição mineral de diversas hortaliças. Bragantia, Campinas, v. 37, n. 1, p. 33-44, 1978.

GARCIA, L. L. C. Absorção de macro e micronutrientes e sintomas de carência de macronutrientes em alface (Lactuca sativa $\mathbf{L}$.) cv. Brasil e Clause's Aurélia. Piracicaba : ESALQ, 1982. 78 p. Dissertação de Mestrado.

GARCIA, L. L. C.; HAAG, H. P.; MINAMI, K.; SARRUGE, J. R. Nutrição mineral de hortaliças: concentração e acúmulo de micronutrientes em alface (Lactuca sativa L.) cv. Brasil e Clause's Aurélia. Anais da Escola Superior de Agricultura Luiz de Queiroz, Piracicaba, v. 39, n. 1, p. 485-504, 1982.

GREWAL, H. S.; ZHONGGU, L.; GRANHAN, R. D. Influence of subsoil zinc on dry matter production, seed yield and distribution of zinc in oilseed rape genotypes differing in zinc efficiency. Plant and Soil, Dordrecht, v. 192, n. 2, p. 181-189, 1997.

IORIO, A. F.; GORGOSCHIDE, L.; RENDINA, A.; BARROS, M. J. Effect of phosphorus, copper, and zinc addition on the phosphorus/copper and phosphorus/zinc interaction in lettuce. Journal of Plant Nutrition, New York, v. 19, n. 3/4, p. 481-491, 1996

LONERAGAN, J. F.; WEBB, M. J. Interactions between zinc and other nutrients affecting the growth of plants. In: ROBSON, A. D. (Ed.). Zinc in soil and plants. Dordrecht : Kluwer Academic, 1993. p. 119-134.

MARSCHNER, H. Mineral nutrition of higher plants. London : Academic, 1995. 889 p.

MARSCHNER, H.; CAKMAK, I. Mechanism of phosphorus induced zinc deficiency in cotton. II. Evidence for impaired shoot control of phosphorus uptake and translocation under $\mathrm{Zn}$ deficiency. Physiologia Plantarum, Copenhagen, v. 68, n. 1, p. 491-496, 1986. 
MOREIRA, M. A. Composição mineral e produção da batateira, em função do uso de fungicidas contendo ou não Zn e do modo de fertilização com $\mathrm{Zn}$. Viçosa : UFV, 1998. 98 p. Dissertação de Mestrado.

PARKER, D. R. Responses of six crop species to solution Zinc $^{+2}$ activities buffered with HEDTA. Soil Science Society of America Journal, Madison, v. 61, n. 1, p. 161167, 1997

RENGEL, Z.; GRANHAM, R. D. Importance of seed $\mathrm{Zn}$ content for wheat growth on Zn-deficient soil. Plant and Soil, Dordrecht, v. 173, n. 2, p. 259-266, 1995.

RESH, H. M. Cultivos hidropónicos. 4. ed. Madrid : Mundi, 1997. 509 p.

SANTOS, I. C.; CASALI, V. W. D.; MIRANDA, G. V. Comportamento de dez cultivares de alface adubadas com composto de lixo urbano. Pesquisa Agropecuária Brasileira, Brasília, v. 33, n. 2, p. 157-161, fev. 1998
STEINER, A. A. The universal nutrient solution. In INTERNATIONAL CONGRESS ON SOILLESS CULTURE, 6., 1984, Lunteren. Proceedings... Lunteren : International Society for Soilless Culture, 1984. p. 633649

WEBB, M. J.; LONERAGAN, J. F. Effect of zinc deficiency on growth, phosphorus concentration, and phosphorus toxicity of wheat plants. Soil Science Society of America Journal, Madison, v. 52, n. 6, p. $1676-1680,1988$

WELCH, R. M.; NORVELL, W. A. Growth and nutrient uptake of barley (Hordeum vulgare L. cv. Herta): studies using an N-(2-hydroxyethyl)ethylenedinitrilotriacetic acid-buffered nutrient solution technique. 1. Role of zinc in the uptake and root leakage of mineral nutrients. Plant Physiology, Rockville, v. 101, n. 2, p. 627-631, 1993. 